\title{
Incidental Mesenteric Paraganglioma: A Case Report and Literature Review
}

\author{
Jennie Granger, Roy Mahapatra, Bushra Hamid, Katherine Gillespie, Matthew Fok, Dale Vimalachandran \\ Colorectal Surgical Department, Countess of Chester Hospital NHS Foundation Trust, Countess of Chester Health Park, Chester, United \\ Kingdom
}

Mesenteric paragangliomas are a rare entity; consequently, only 12 cases have been reported to date. Although considered benign and often found incidentally, they have the potential to metastasize and are an important diagnosis to consider for patients with a mesenteric tumor. We report the case of a 71-year-old woman who was found on magnetic resonance imaging to have an incidental, large, complex, intra-abdominal mass that had initially been misdiagnosed as ovarian in origin. She underwent an open resection of the tumor and adjacent small bowel with no perioperative complications; histology confirmed the mass to be a mesenteric paraganglioma. This case report highlights several important key issues regarding paraganglioma, including diagnosis, imaging, genetic testing, and surgical resection. The results of a literature review are also discussed.

\section{Keywords: Paraganglioma; Intra-abdominal tumor; Abdominal surgery}

\section{INTRODUCTION}

A mesenteric paraganglioma is a rare entity. In fact, we were able to find only 12 cases in the literature to date. Although paraganglioma are considered benign and are often found incidentally, they have the potential to metastasize. Thus, they are an important diagnosis to consider for patients with a mesenteric tumor.

We report the case of a 71-year-old woman with a large, complex, intra-abdominal mass that was found on magnetic resonance imaging (MRI) and had initially been misdiagnosed as ovarian in origin. She underwent an open resection of the tumor and adjacent small bowel, and no perioperative complications occurred. Histology confirmed the mass to be a mesenteric paraganglioma. This case report highlights several important issues regarding paragangliomas. The results of a literature review are also discussed.

Received: May 11, 2017 - Accepted: September 26, 2017

Correspondence to: Dale Vimalachandran, FRCS

Colorectal Surgical Department, Countess of Chester Hospital NHS

Foundation Trust, Countess of Chester Health Park, Liverpool Road, Chester

CH2 1UL, United Kingdom

Tel: +44-10244-365475, Fax: +44-10344-369292

E-mail: Dale.vimalachandran@nhs.net

(C) 2017 The Korean Society of Coloproctology

This is an open-access article distributed under the terms of the Creative Commons Attribution NonCommercial License (http://creativecommons.org/licenses/by-nc/4.0) which permits unrestricted noncommercial use, distribution, and reproduction in any medium, provided the original work is properly cited.

\section{CASE REPORT}

We report the case of a 71-year-old woman who initially underwent outpatient MRI of the lumbar spine for an abnormal gait and sciatica when she was found to have an incidental, large pelvic mass (Fig. 1). Her past medical history included an appendectomy and ovarian cystectomy, osteoarthritis, and peripheral neuropathy. Following MRI, she was referred for an emergency outpatient gynecological opinion, at which time a transabdominal ultrasound scan confirmed the presence of a $9 \times 5.6 \times 10-\mathrm{cm}$ complex cystic mass posterior to the bladder and superior to the fundus of the uterus (Fig. 2). The ovaries could not be identified, and blood tests, including tests to determine full blood count, kidney function, liver function, and CA125 and carcinoembryonic antigen levels, were all normal. Despite a large palpable abdominal mass, she remained relatively asymptomatic, complaining of some lower abdominal discomfort and urinary incontinence controlled with a pessary. In light of the normal CA125 and large pelvic mass, she was booked for an urgent diagnostic laparoscopy, bilateral salpingo-oophorectomy, and excision of the large pelvic mass. Laparoscopy revealed the mass to be arising from the small bowel mesentery, and the only procedure performed was peritoneal washing, which did not show any significant abnormality microscopically. A follow-up computed tomography (CT) scan of her chest, abdomen, and pelvis was done, and her condition was discussed at the colorectal multidisciplinary 


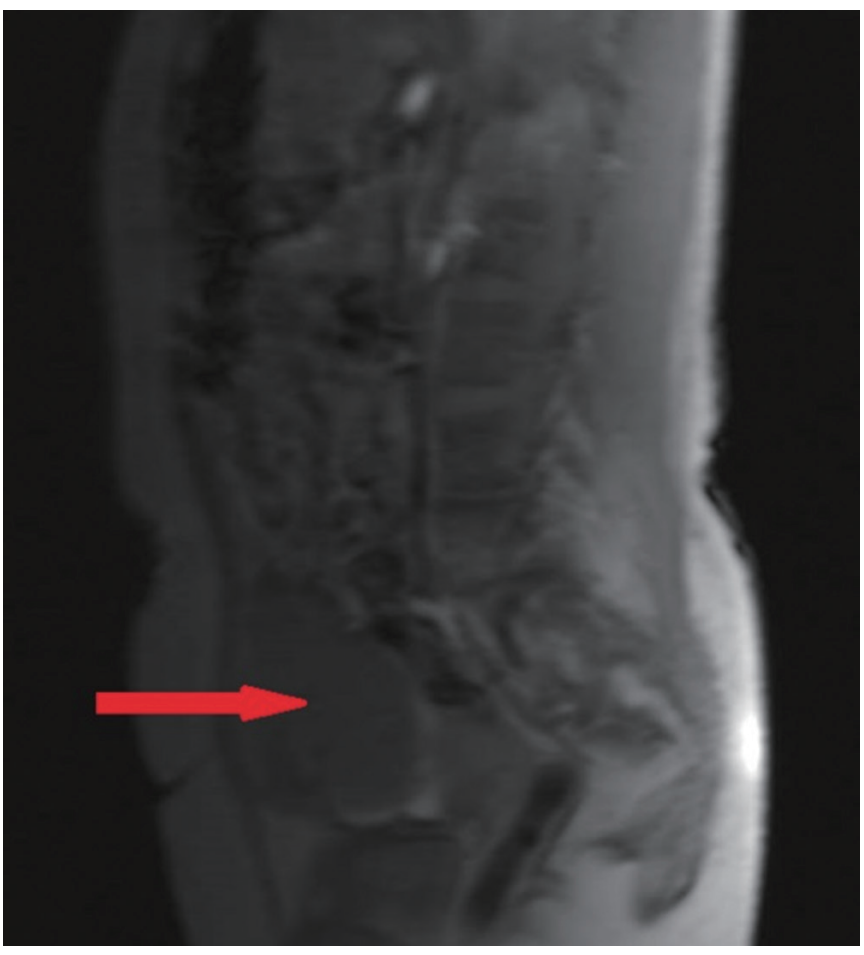

Fig. 1. Magnetic resonance imaging of the lumbar spine which on a scout view shows an impression of a large pelvic mass (red arrow). team (MDT) meeting.

The CT scan revealed a well-encapsulated enhancing mass with multiple septations and locules measuring $8.6 \mathrm{~cm} \times 9 \mathrm{~cm} \times 7.5$ $\mathrm{cm}$ (Fig. 3). Neither invasion into adjacent structures nor any associated lymphadenopathy was observed. Two indeterminate low attenuation lesions without any ascites or peritoneal deposits were found within the liver. She went on to have a lower midline laparotomy and excision of her mass. At the time of operation, dissec-

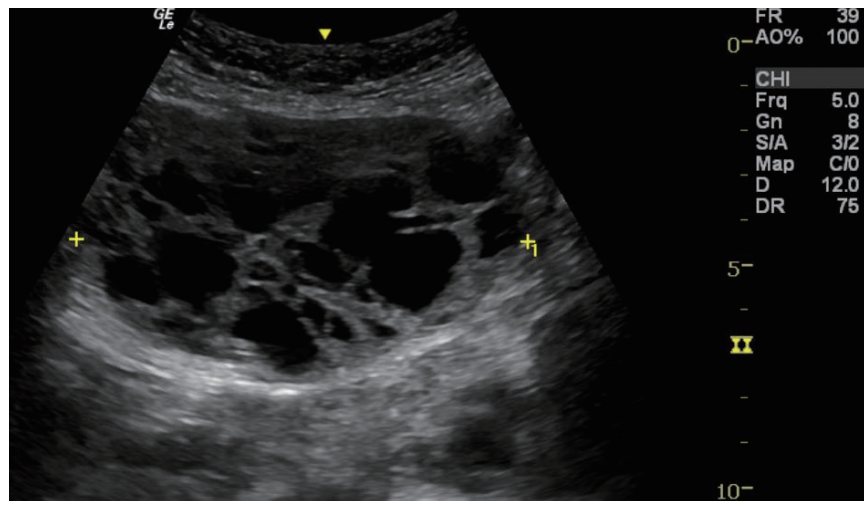

Fig. 2. Ultrasound scan of the abdomen and pelvis showing a large, complex, cystic mass posterior to the bladder and above the fundus of uterus, with neither ovary being seen separate from the mass.
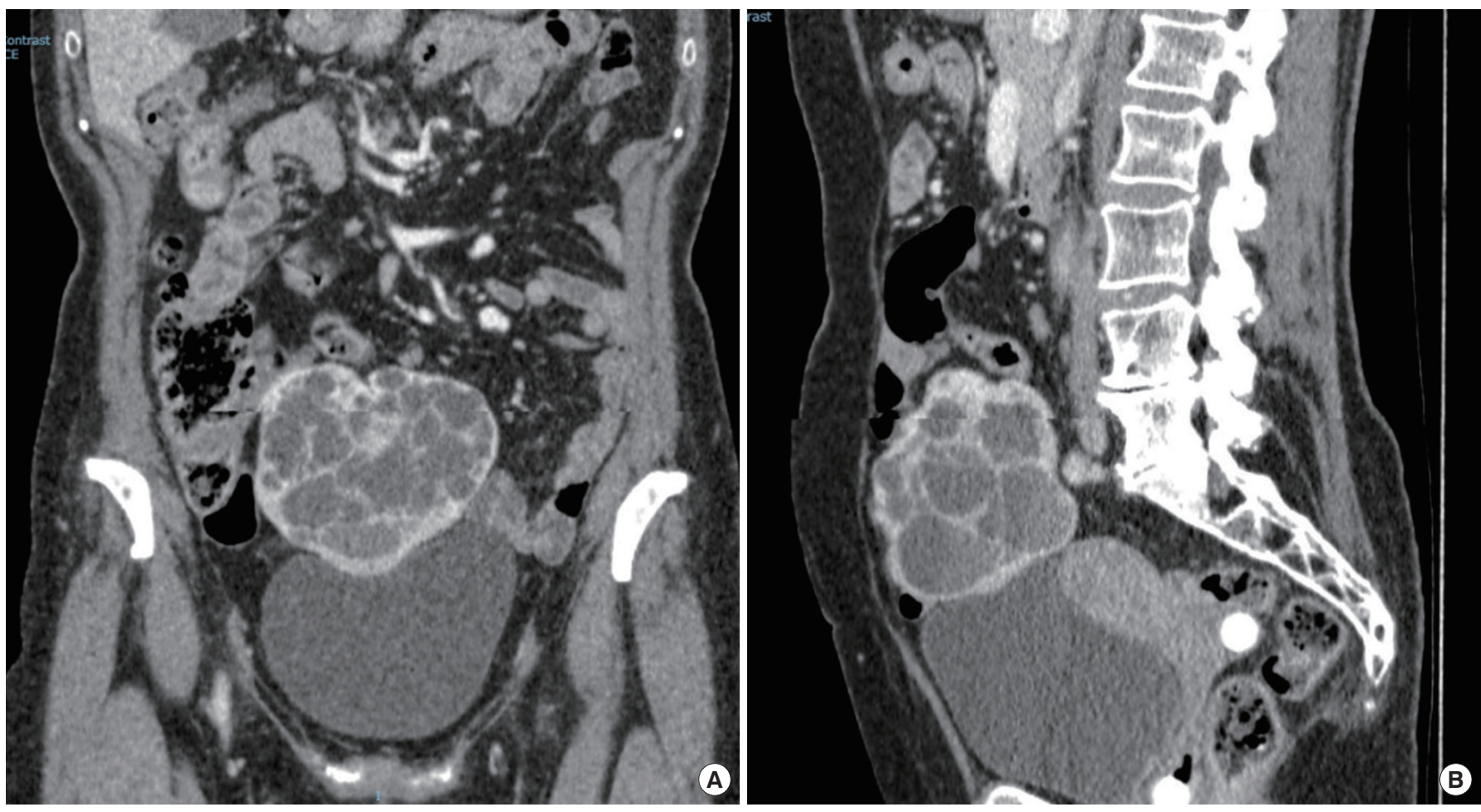

Fig. 3. Coronal (A) and sagittal (B) computed tomographic scans of the abdomen showing a large mass measuring $8.6 \mathrm{~cm} \times 9 \mathrm{~cm} \times 7.5 \mathrm{~cm}$ with multiple septations and locules. 
Volume 33, Number 5, 2017

Ann Coloproctol 2017;33(5):197-200 Coloproctology

tion of the mass from the mesentery was unsuccessful, and complete excision required the removal of approximately $50 \mathrm{~cm}$ of the small bowel, followed by a hand sewn anastomosis with interrupted 3-0 polydioxanone sutures. Her recovery was uneventful, and she was discharged.

Macroscopically, the mass, which measured $105 \mathrm{~mm} \times 90 \mathrm{~mm}$ $\times 75 \mathrm{~mm}$, was within the mesentery of the small bowel and had a brown, lobulated external surface and a multiloculated, cystic cut surface. The small bowel itself was unremarkable. Microscopic examination showed a well-circumscribed neoplasm composed of nests and trabeculae of polygonal cells with moderate amounts of eosinophilic cytoplasm (Fig. 4). Immunohistochemical staining showed strong diffuse staining with chromogranin (Fig. 5A), synaptophysin (Fig. 5B), CD 56, and vimentin, with patchy positivity

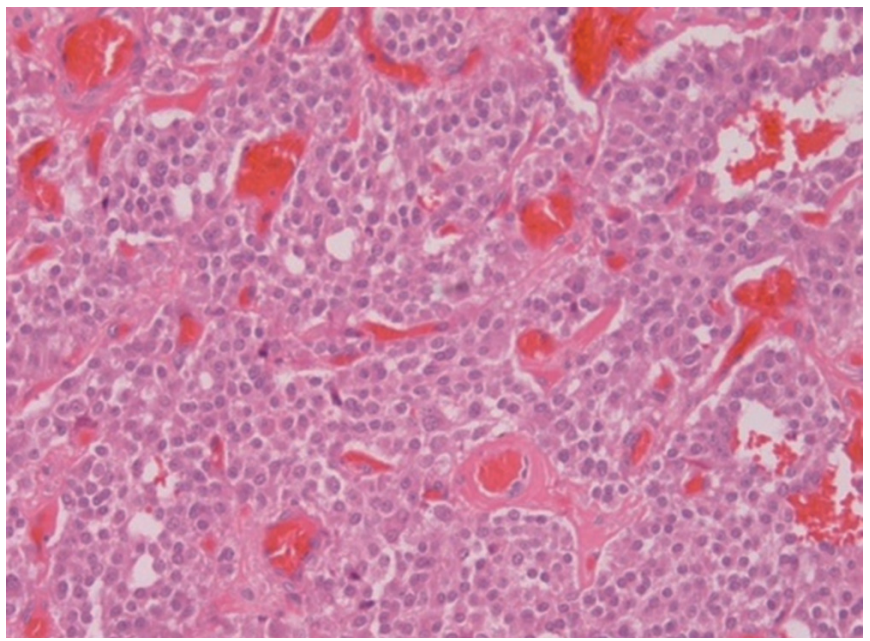

Fig. 4. Microscopic examination showed a well-circumscribed neoplasm composed of nests and trabeculae of polygonal cells with moderate amounts of eosinophilic cytoplasm.

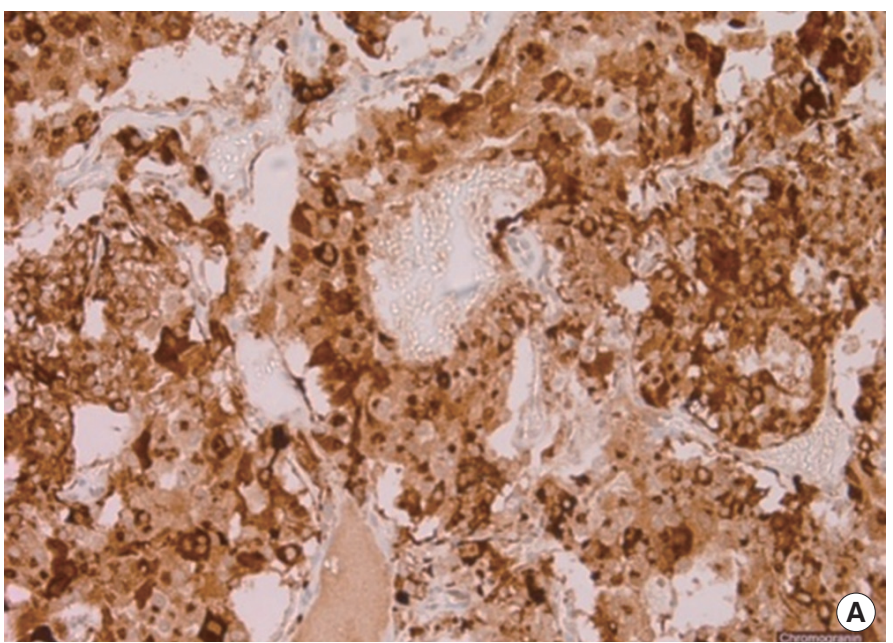

for S100 protein and BcL2. AE1/3, CD34, CD117, desmin, MNF116, and podoplanin were negative. MIB-1 showed less than $1 \%$ positivity. These findings are consistent with a diagnosis of a paraganglioma.

During her postoperative follow-up, liver MRI with primovist revealed a $24-\mathrm{mm}$ hypovascular lesion that could not be further characterized. Her case was discussed at the regional neuroendocrine tumor MDT, and a Gallium 68 Dotanoc positron emission tomography (PET) CT scan was recommended; the scan showed no evidence of uptake in the liver and no evidence of disease elsewhere. She continues to remain well one year postoperatively and is under yearly surveillance with CT.

Written informed consent was obtained from the patient.

\section{DISCUSSION}

Paragangliomas are rare tumors of neural crest origin and may arise from any area with sympathetic or parasympathetic paraganglia [1]. Although these tumors are on the whole considered benign, they can metastasize. However, a huge variation in the incidence of metastases is reported in the literature (3\%-50\%) [2]. Importantly, paragangliomas are often found within the adrenal medulla and may give rise to pheochromocytomas; however, up to $10 \%$ of these tumors are found in extra-adrenal sites, with a predilection to the abdomen. Those tumors within the abdomen are often located adjacent to the aorta and particularly the area corresponding to the organ of Zuckernadl beginning cranial to the superior mesenteric artery and extending to the aortic bifurcation [1]. To our knowledge, 12 cases of mesenteric paragangliomas have been reported [1].

The rarity of paragangliomas is typified by the limited number of cases reported in the literature, with little guidance being available regarding their management. This rarity is further reflected in published cases with delayed diagnosis. Furthermore, as the

Fig. 5. Immunohistochemical staining showed strong diffuse staining with chromogranin (A) and synaptophysin (B).

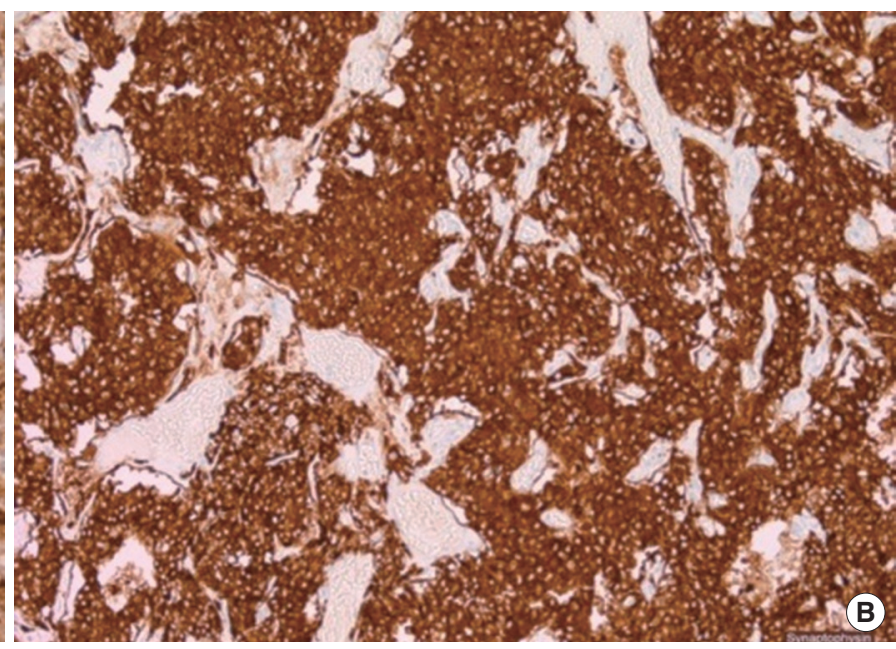


majority of intra-abdominal paragangliomas are both benign and nonfunctional, they are often found incidentally or following symptoms related to their size. Very rarely, intra-abdominal paragangliomas are functional, producing clinically significant levels of catecholamines and thus presenting with paroxysmal hypertension and the classical triad of symptoms associated with pheochromocytomas (palpitations, headaches, and profuse sweating) $[1,3,4]$.

In view of the low incidence of reported cases, the pathogenesis and the natural history of paragangliomas are poorly understood. Paragangliomas have a hereditary association in as high as $50 \%$ of cases $[1,3]$. Familial paragangliomas display an autosomal dominant inheritance and incomplete penetrance. Furthermore, hereditary cases have also been associated with multiple endocrine neoplasia 2, von Hippel-Lidau disease, Carney triad, and neurofibromatosis type 1; consequently, genetic testing should be considered for all patients diagnosed with paragangliomas [1].

Imaging, usually in the form of a CT scan, plays a key role in the preoperative planning for the resection of a paraganglioma [4]. Other considerations should be given to 131I-metaiodobenzlguanidine scintigraphy in the diagnosis of a functional paraganglioma $[1,4]$. Moreover, fluorodeoxyglucose-PET scanning can be useful in evaluating the presence of metastatic disease [5].

Surgical resection resulted in good outcomes for the 12 reported cases of mesenteric paragangliomas [1]. As with our patient, those found in the small bowel mesentery usually required resection of adjacent bowel, which was mainly due to the proximity of the mass to the bowel or to the risk of devascularization of the bowel. A single case report described a laparoscopic resection of a small paraganglioma found within the mesentery of the terminal ileum; in all other reports, including our own, the resections were performed through a midline laparotomy. None of the reported cases mentions any evidence of reoccurrence of disease following surgi- cal resection (range of follow-up: 8-32 months) [1, 4].

In conclusion, our case report highlights several key clinical points: First, the diagnosis of intra-abdominal paragangliomas is often difficult and/or incidental. Second, imaging must be considered pre- and postoperatively in order to exclude the possibility of metastatic disease. Third, genetic testing should be considered in any patients diagnosed with a paraganglioma. Finally, surgical resection yields a good prognosis. With this report, we aim to raise awareness that a paraganglioma should be included in the differential diagnosis of any mesenteric tumors.

\section{CONFLICT OF INTEREST}

No potential conflict of interest relevant to this article was reported.

\section{REFERENCES}

1. Fujita T, Kamiya K, Takahashi Y, Miyazaki S, Iino I, Kikuchi H, et al. Mesenteric paraganglioma: report of a case. World J Gastrointest Surg 2013;5:62-7.

2. Fliedner SM, Lehnert H, Pacak K. Metastatic paraganglioma. Semin Oncol 2010;37:627-37.

3. Trombetta M, Silverman J, Colonias A, Lee V, Mohanty A, Parda D. Paraganglioma: a potentially challenging tumor. Oncology (Williston Park) 2008;22:341-3, 347, 350; discussion 350-2.

4. Chetrit M, Dubé P, Royal V, Leblanc G, Sideris L. Malignant paraganglioma of the mesentery: a case report and review of literature. World J Surg Oncol 2012;10:46.

5. Vázquez-Quintana E, Vargas R, Pérez M, Porro R, Gómez Duarte C, Tellado M, et al. Pheocromocytoma and gastrointestinal bleeding. Am Surg 1995;61:937-9. 